\title{
Understanding Innovation as Change of Value Systems
}

\author{
John S. Gero ${ }^{1}$ and Udo Kannengiesser ${ }^{2}$ \\ ${ }^{1}$ Krasnow Institute for Advanced Study and Volgenau School of Information Technology \\ and Engineering, George Mason University, USA, and University of Technology, \\ Sydney, Australia \\ ${ }^{2}$ NICTA, Australia, and School of Computer Science and Engineering, University of New \\ South Wales, Sydney, Australia \\ john@johngero.com, udo.kannengiesser@nicta.com.au
}

\begin{abstract}
This paper presents a view of innovation as a process that changes value systems of producers and adopters of creative design ideas. Value systems comprise interpretations of the function and behaviour of an artefact, encapsulated in a producer's or adopter's situation. Changes in these value systems can be induced using distinct classes of processes. The paper shows that innovation requires changes in the encapsulated value systems of both producers and adopters, driven by their interactions with the artefact and with each other. This provides the basis for a computational, agent-based framework for testing models of innovation.
\end{abstract}

Keywords: Situations, Function-Behaviour-Structure Ontology, Agent-Based Systems.

\section{Introduction}

Innovation is commonly understood as a process that realises creative design ideas, i.e. turns them into practice. It is widely accepted that this process is critical, no matter whether the creative idea relates to the design of products, processes, services or organisations. However, the focus of most research in computer-aided innovation has been on idea generation rather than idea realisation. This is partly due to a view of innovation as an ancillary, downstream activity that is beyond the traditional scope of designing.

Both idea generation and idea realisation may occur in various design domains and across various life-cycle stages [1-3]. In all cases, they involve a producer and an adopter of the creative design idea. The central hypothesis of this paper is that innovation is a process that changes the value systems of both producers and adopters. They are encapsulated in the situation that an agent constructs using its individual view of the world it interacts with. Section 2 represents value systems using the function-behaviour-structure (FBS) ontology, and situations using the idea of three interacting worlds [4]. Section 3 characterises changes of value systems at this ontological level. Section 4 presents a set of classes of processes that can induce changes of value systems of adopters and producers. Section 5 proposes a conceptual 
architecture for developing agent-based systems that can be used for simulating and testing models of innovation. Section 6 concludes the paper with some suggestions for future research.

\section{Design Properties, Value Systems and Situations}

The FBS ontology provides all the constructs needed to represent the properties of an artefact [4]:

- Function (F) of a design object is its teleology ("what it is for"). An example is the function "to wake someone up" that humans generally ascribe to the behaviour of an alarm clock.

- Behaviour (B) of a design object is the attributes that can be derived from its structure ("what it does"). An example of a physical artefact is "weight", which can be derived directly from the product's structure properties of material and geometry.

- Structure (S) of a design object is its components and their relationships ("what it consists of"). For physical artefacts, it comprises geometry, topology and material.

Humans construct relationships between function, behaviour and structure through experience and through the development of causal models based on interactions with the object. Function is ascribed to behaviour by establishing a teleological connection between the human's goals and measurable effects of the object. Behaviour is causally related to structure, i.e. it can be derived from structure using physical laws or heuristics. This may require knowledge about external effects and their interaction with the object's structure. There is no direct relationship between function and structure.

Designing deals primarily with models of the object rather than with the object itself. On the other hand, most downstream activities of the product life cycle operate on the physical product or its components and materials. However, the FBS ontology does not distinguish between different embodiments of an object. All objects can be captured as function, behaviour and structure, no matter whether they are embodied in a symbolic (e.g., computational) environment or in the physical world. This makes the FBS ontology an appropriate basis for describing design objects at any stage in their life cycle.

Value systems can be defined in terms of design properties that relate to the notion of usefulness for stakeholders in the life cycle. In the FBS view of the world, this includes function, as it captures the usefulness of design objects by definition. It also includes behaviour as a measure for the potential of a design object to achieve the function. Structure is not a part of a value system, because it is not related to usefulness in a strict sense. However, structure can be viewed as an implicit value system, since its design is driven by intended function and behaviour.

Value systems are encapsulated in situations that are different for every agent. The process that produces situations is called situatedness [5], and can be modelled as the interaction between three worlds [4], as shown in Fig. 1. Situations are the carriers of the value systems. As such they produce expectations which guide interpretations. 


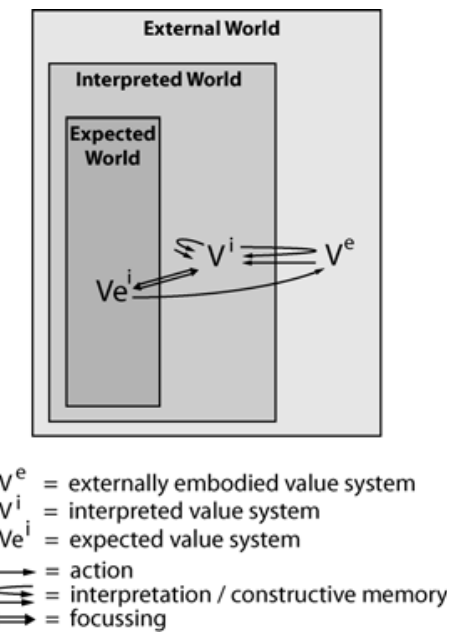

Fig. 1. Value systems (V) encapsulated in a situation (interpreted and expected worlds), interacting with externally embodied value systems

The external world contains symbolic or physically embodied value systems made available for interpretation. The value systems may be explicit or implicit. Explicit value systems include function or behaviour, as outlined earlier. Implicit value systems are externally embodied structures that afford certain behaviour and function.

The interpreted world provides an environment for analytic and associative activities, related to current and previous value systems. It uses interpretation and memory processes, both of which are represented in Figure 1 using "push-pull" arrows to account for their dynamic character as an interaction of data-push and expectation-pull [6]. As a result, interpretations and memories can change over time, which then affect subsequent interpretations and memories. It is in this dynamic world where implicit value systems may become explicit, i.e. where structure, once interpreted, is turned into behaviour that then may have functions attributed to it.

The expected world (within the interpreted world) forms goals through focussing on parts of the interpreted value systems, and predicts the effects of actions to modify the (explicit or implicit) value systems in the external world.

A situation in this model can be viewed as a snapshot of the interpreted and expected worlds at a particular point in time. The situation changes as a result of interactions between the three worlds (and "push-pull" interactions within the interpreted world). In turn, changes in the situation set up expectations that drive both interpretations and what situations can be constructed in the future. This means that the same external world with different situations produces different interpreted worlds and then expected worlds. As the situation changes, the encapsulated value systems change accordingly.

\section{Characterising Changes of Value Systems}

Innovation is claimed to change the value systems of adopters. We can classify changes of (explicit) value systems depending on whether they affect function or behaviour. 


\subsection{Changes at the Function Level}

At the function level, we distinguish two classes of changes of value systems: ${ }^{1}$

1. Sustaining changes: allow for improved practices while sustaining the adopter's existing needs and criteria for assessing improvements. These effects are often expressed using qualitative, comparative statements such as "more efficient", "more convenient" and "simpler".

2. Disruptive changes: enable the emergence of new practices and needs, setting up new benchmarks for any innovative design outcome that may follow. These changes may lead to the creation of new markets and the destruction of existing markets [7].

\subsection{Changes at the Behaviour Level}

At the behaviour level, we distinguish two classes of changes of value systems:

1. Homogeneous changes: affect behaviour in a quantitative way, without introducing new classes of behaviour. The changes may be small-scale (e.g., a new car design with a $5 \%$ reduction in petrol consumption) or large-scale (e.g., a new car design with a $40 \%$ reduction in petrol consumption).

2. Heterogeneous changes: affect behaviour in a qualitative way, i.e. some classes of behaviour are added to and/or removed from the previous ones. For example, a change from "petrol consumption" to "rapeseed oil consumption" can be characterised as heterogeneous.

There is a loose connection between this classification and the one for changes at the function level. Disruptive changes (at the function level) usually require heterogeneous changes (at the behaviour level). In some cases, large-scale homogeneous changes can also lead to disruptive changes. However, these connections are far from fixed, as value systems are encapsulated in a situation that governs the construction of all values and their relationships.

\section{Inducing Change of Value Systems}

How are changes of value systems brought about? Given that value systems are encapsulated in a situation, this question is not the same as "how can we produce designs with novel function or behaviour". Exploring how value systems can be changed requires an understanding of the interactions between producers and adopters that can affect situations. These interactions can be viewed as part of a two-way communication process in which producers aim to influence adopters [9] and adopters provide direct or indirect feedback to producers.

\subsection{Changing Adopter Situations}

There are three ways of inducing change in adopter situations:

\footnotetext{
${ }^{1}$ We have borrowed the names of the two classes from Bower and Christensen's [8] notions of "sustaining" and "disruptive" technologies.
} 
1. Social influence: is based on presenting explicit value systems $\left(\mathrm{V}^{\mathrm{e}}\right)$ that the adopter accepts without much self reasoning. Examples include product marketing, peer pressure (which may be part of a "viral marketing" strategy), and product tests or recommendations from trusted authorities (e.g., newspapers, consumer groups and industry associations).

2. Affordance: is an adopter's interpretation process that makes implicit value systems $\left(\mathrm{V}^{\mathrm{e}}\right)$ explicit in the way intended or unintended by the producer. This process can be characterised as entailing a small amount of interaction between data-push and expectation-pull in this process. In other words, the data presented is consistent with the adopter's grounded expectations (or conventions) [10]. Hence, affordance is very much correlated with the notion of sustaining changes. For example, a mobile phone of reduced physical dimensions affords "better portability", sustaining the adopter's existing needs.

3. Emergence: comprises interpretation and constructive memory processes with more significant amounts of interaction between the adopter's expectations and the (explicit or implicit) value system $\left(\mathrm{V}^{\mathrm{e}}\right)$. It can be viewed as a form of "unintended" innovation, based on mechanisms such as analogical reasoning. An example of an emergent innovation is Scotch Tape, whose initial function "to mend books" was transformed by the end user into a number of different functions, such as "to wrap packages" and "to curl hair" [11]. Other examples of emergent innovations, according to Redström [12], include the record player (originally a sound reproduction device, turned into a musical instrument by DJs) and the skateboard (originally a children's scooter, but the handles accidentally fell off and children experimented with it).

\subsection{Changing Producer Situations}

In general, it is advantageous for producers to monitor and analyse changes in adopters' value systems. Original producers use this information to refine their innovative designs. Current and future competitors use the same information to identify the key technologies and assess their own capabilities for entering the market with similar designs but improved characteristics such as lower cost or better styling. In addition, producers may generate new creative design ideas based on their analyses of adopters' value systems. These ideas may target existing markets or the creation of new markets.

What is common in all of these cases is that the producers' value systems can change based on changes in their situations. There are three ways of inducing change in producer situations:

1. Direct feedback: may be available through questionnaires, customer support, complaint forms or other feedback provided by adopters. This feedback represents explicit value systems $\left(\mathrm{V}^{\mathrm{e}}\right)$, and can be viewed as a direct form of communication from adopters to producers. Participatory design methodologies integrate explicit user feedback in the process of designing, aiming to identify opportunities for improvement and novel ideas in the early stages of innovation.

2. Observations: can be seen as a form of indirect feedback, based on studying the intentional use of a design by adopters. A good example is Scotch Tape, mentioned earlier. Here, the various modes of use invented by the adopters (wrapping 
packages, curling hair, etc.) were observed (and interpreted as functions) by the original producers. They then refined their product by creating a range of product variations that were adapted to the specific functions: "As a result, 3M [the company of the producers] came out with a hair-setting Scotch Tape, a medical Scotch Tape used for binding splints, a reflective Scotch Tape for roads, and so on" [13]. This new range of products can be seen as a consequence of changes in producers' value systems.

3. Emergence: generates value systems that are novel with respect to both producers and adopters. They result from the producers' interpretation and constructive memory processes and their interactions with the adopters' value systems. For example, when Sony introduced the Walkman they explicitly changed the size of portable music devices by eliminating the loudspeaker and replacing it with earbuds. However, in doing so they produced an emergent value: listening to music became a private rather than a public activity and consequentially spawned an entire industry.

The products resulting from the changes in the producers' value systems can again lead to innovation, by subsequently changing the adopters' value systems. This shows that innovation involves a set of interactions between producers and adopters, which can be viewed as a process of reflective conversation [12].

\section{Towards Agent-Based Simulations of Innovation}

We can synthesise the concepts developed in the previous Sections into an agentbased framework. This can be used to develop computational systems that allow simulating theories of innovation and testing whether they are consistent with the understanding of innovation outlined in this paper. Fig. 2 shows the conceptual architecture for such a system.

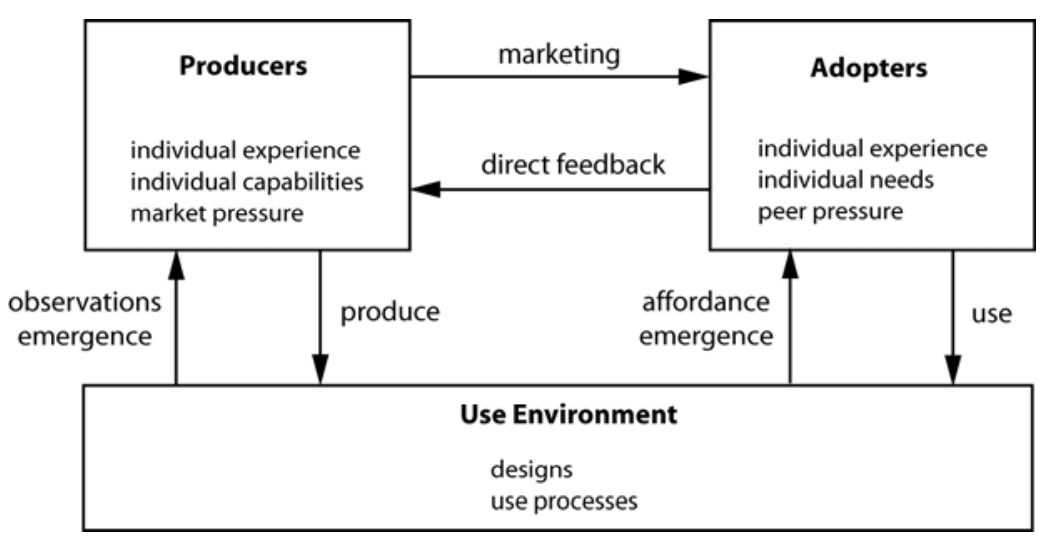

Fig. 2. Architecture for an agent-based social system for simulating design innovation 
The architecture consists of three components:

- Producers: are design agents that have the capacity to transform explicit value systems (i.e., function and behaviour) into implicit value systems (i.e., structure) embodied as products. A number of factors influence their transformations, including individual experience and capabilities, and requirements imposed by perceived market pressures.

- Adopters: are agents (that may or may not be design agents) with the capacity to transform implicit value systems (i.e., structure) into explicit value systems (i.e., function and behaviour) that determine if and how they use the product. A number of factors influence their transformations, including individual experience and needs, and perceived peer pressure.

- Use environment: is the "actual" or simulated world of products and processes using these products. This environment is shared and accessible by both producers and adopters.

Agent-based social simulations based on this architecture can be used in the study of two notions of innovation: (1) innovation as an individual process, changing value systems related to a single artefact; and (2) innovation as a set of processes, changing a collection of value systems related to multiple artefacts.

\subsection{Studying Innovation as an Individual Process}

Two competing hypotheses can be tested:

1. The Planning Hypothesis: assumes innovation as a deterministic process, where producers successfully apply strategies that induce the desired change of adopters' value systems. Specifically, producers are expected to generate designs with appropriate affordances [14] and appropriate marketing methods. Emergence ("unintended innovation") does not play a role here, since all innovations are assumed to be intended by the producers. This also implies that innovation is a unidirectional, "one-shot" process from producers to adopters. The Planning Hypothesis can be formulated without using the notion of encapsulated value systems, because of its simplified view of situations.

2. The Reflection Hypothesis: assumes innovation as a phenomenological process that involves unexpected changes of value systems of both adopters and producers. Emergence plays a major role here, as it provides the potential for changing the situation of an agent. Under this hypothesis, innovation is assumed to frequently involve several cycles of bidirectional producer-adopter interactions, consistent with Schön's idea of reflective conversation. The notions of value systems and their encapsulating situations are crucial elements of the Reflection Hypothesis.

Based on the examples we have reported from design literature, we expect that simulations will be likely to support the Reflection Hypothesis.

\subsection{Studying Innovation as a Set of Processes}

Two competing hypotheses can be tested:

1. The Static System Hypothesis: assumes that the effects of innovation contribute additively to an otherwise static set of value systems in a society of adopters and 
producers. Every individual innovation that is adopted does not modify the adopters' expectations for evaluating future and past designs. Similar to the Planning Hypothesis, the Static System Hypothesis does not need the notion of encapsulated value systems in the way presented in this paper.

2. The Cultural Evolution Hypothesis: assumes innovation as a sequence of situated acts, where every change of a value system generates new expectations for evaluating future and past designs. This leads to continuous changes in the collective value systems of the society of producers and adopters, leading to emergent patterns that can be interpreted as cultural evolution. This hypothesis requires encapsulated value systems as its central underlying constructs.

We expect that simulations will be likely to confirm the Cultural Evolution Hypothesis.

\section{Conclusion}

This paper argues that innovation involves not just the adoption of new products and processes but also involves changing the values of the adopters and the producers in such a way that all further evaluations of past and future products and processes are changed. The paper describes how the notions of situatedness and situations provide a basis for the modelling of innovation as a change in the value systems. Such a change of values models Schumpeter's "creative destruction" theory of innovation [7]. We have outlined an architecture for agent-based social systems that can be used for testing this theory and related hypotheses.

Future work may develop various extensions of our model of innovation. We suggest that they should cover three aspects that we have discussed only briefly in this paper. They include the effects of social ties, amplifiers and gatekeepers among both producers and adopters. Social ties refer to formal or informal groups of agents. Amplifiers include agents or groups of agents with high visibility or reputation among their peers. Gatekeepers are agents or groups of agents with the normative authority to allow or reject specific designs to enter a society of producers or adopters. This authority may be given explicitly (by laws or regulations; e.g., certification authorities) or implicitly (by opinion leadership; e.g., trade magazines or market leaders). Describing these aspects with appropriate parameters, and including them in a computational model, allows simulating innovation processes under varying conditions. The insights resulting from these simulations can be useful for innovators and innovation policy makers alike, by better informing their innovation strategies with respect to different social or market conditions.

\section{Acknowledgments}

This work is an outgrowth of research supported by a grant from the Australian Research Council, grant no: DP0559885.

NICTA is funded by the Australian Government as represented by the Department of Broadband, Communications and the Digital Economy and the Australian Research Council through the ICT Centre of Excellence program. 


\section{References}

1. Archer, B.L.: Technological Innovation: A Methodology. Inforlink, London (1971)

2. Roozenburg, N.F.M., Eekels, J.: Product Design: Fundamentals and Methods. Wiley, New York (1995)

3. Buijs, J.: Modelling Product Innovation Processes, from Linear Logic to Circular Chaos. Creativity and Innovation Management 12(2), 76-93 (2003)

4. Gero, J.S., Kannengiesser, U.: The Situated Function-Behaviour-Structure Framework. Design Studies 25(4), 373-391 (2004)

5. Clancey, W.J.: Situated Cognition: On Human Knowledge and Computer Representations. Cambridge University Press, Cambridge (1997)

6. Gero, J.S.: Constructive Memory in Design Thinking. In: Goldschmidt, G., Porter, W. (eds.) Design Thinking Research Symposium: Design Representation, pp. 29-35. MIT, Cambridge (1999)

7. Schumpeter, J.A.: Capitalism, Socialism and Democracy. Harper, New York (1942)

8. Bower, J.L., Christensen, C.M.: Disruptive Technologies: Catching the Wave. Harvard Business Review 73(1), 43-53 (1995)

9. Crilly, N., Good, D., Matravers, D., Clarkson, P.J.: Design as Communication: Exploring the Validity and Utility of Relating Intention to Interpretation. Design Studies 29(5), 425457 (2008)

10. Brown, D.C., Blessing, L.T.M.: The Relationship between Function and Affordance. In: ASME 2005 Design Theory and Methodology Conference, Long Beach, CA, paper no. DETC2005-85017 (2005)

11. Schön, D.A.: The Reflective Practitioner: How Professionals Think in Action. Harper Collins, New York (1983)

12. Redström, J.: RE: Definitions of Use. Design Studies 29(4), 410-423 (2008)

13. Schön, D.A., Bennett, J.: Reflective Conversation with Materials. Bringing Design to Software. ACM, New York (1996)

14. Maier, J.R.A., Fadel, G.M.: Affordance Based Design: A Relational Theory for Design. Research in Engineering Design 20(1), 13-27 (2009) 